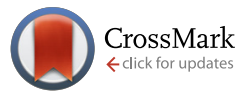

Cite this: Lab Chip, 2015, 15, 1742

Received 21st November 2014, Accepted 4th February 2015

DOI: 10.1039/c4lc01376j

www.rsc.org/loc

\section{Direct laser writing of $\mu$-chips based on hybrid C-Au-Ag nanoparticles for express analysis of hazardous and biological substances $\dagger$}

\author{
M. Y. Bashouti, ${ }^{\text {a }}$ A. Manshina, ${ }^{\text {b } A . ~ P o v o l o t c k a i a, ~}{ }^{c}$ A. Povolotskiy, ${ }^{b}$ A. Kireev, ${ }^{b}$ \\ Y. Petrov, ${ }^{d}$ M. Mačković, ${ }^{e}$ E. Spiecker, ${ }^{e}$ I. Koshevoy, ${ }^{b}$ S. Tunik ${ }^{b}$ and S. Christiansen ${ }^{\text {af }}$
}

Detection and identification of low concentrations in small volumes of hazardous and biological substances are currently important to address challenges in environmental monitoring, homeland security and medicine. Recently, different groups have developed devices based on metal nanoparticles (NPs) with the capacity to detect low concentrations of substances. ${ }^{1}$ However, devices associated with NPs require two stage development: (i) synthesis of NPs and (ii) integration of the NPs into devices. Even though the synthesis of NPs is well established (especially for Au and Ag) and different integrating approaches of NPs into devices have been demonstrated (such as Layer-by-Layer and drop casting, optical and nano lithography, direct laser writing of optofluidic and multifunctional microfluidic chips, laser ablation of Si wafer surfaces, followed by coating with metal thin films such as gold etc.), ${ }^{2}$ the state-of-the art sensors/devices still suffer from a lack of control over parameters. Moreover,

\footnotetext{
${ }^{a}$ Max-Planck Institute for the Science of Light, Erlangen D-91058, Germany. E-mail: Muhammad.Bashouti@mpl.mpg.de

${ }^{b}$ Saint-Petersburg State University, Department of Chemistry, Saint-Petersburg 198504, Russia

${ }^{c}$ Saint-Petersburg State University, Department of Physics, Saint-Petersburg 198504, Russia

${ }^{d}$ Saint-Petersburg State University, Interdisciplinary Resource Center for Nanotechnology, Saint-Petersburg 198504, Russia

${ }^{e}$ Universität Erlangen-Nürnberg, Center for Nanoanalysis and Electron Microscopy (CENEM), Department of Materials Science and Engineering, Erlangen D-91058, Germany

${ }^{f}$ Institute of Nanoarchitectures for Energy Conversion, Helmholtz-Centre Berlin (HZB), Berlin, D-14109, Germany

$\dagger$ Electronic supplementary information (ESI) available: Supporting information for this article is given via a link at the end of the document. See DOI: 10.1039/ c4lc01376j
}

chemical functionalities on the NP surfaces are not stable over time and react with $\mathrm{H}_{2} \mathrm{O} / \mathrm{O}_{2}$ in ambient conditions and impair the device performance. ${ }^{1 e}$

Here, we propose a new method for the fabrication of micro-chip devices (for short $\mu$-devices) based on nanoalloys of $\mathrm{Au}-\mathrm{Ag}$ NPs that are simultaneously synthesized by direct laser writing and embedded in a carbonaceous matrix (hereinafter, $\mathrm{C}-\mathrm{Au}-\mathrm{Ag}$ NPs). It should be noted that the suggested process is photoinduced that allows avoiding side effects of thermal phenomena (delocalization of the deposition process, thermal decomposition, etc.). The C-Au-Ag NPs provide high stability and new functionalities of combined properties of the metals and the carbon that opens the door for new applications. ${ }^{3}$ For example, a core of gold-silver nanoalloys coated with a carbon shell demonstrates excellent efficiency as a Raman tag for quantitative immunoassays and high catalytic activity. ${ }^{3}$ However, the preparation of metal NPs or nanoalloys in a carbonaceous matrix is complicated, time consuming and requires the use of dangerous chemical compounds and chemical equipment. ${ }^{4}$ In our case, we present a fast and coherent Laser-Induced liquid phase Deposition (LID) method for direct writing of $\mu$-chips based on NPs from solutions on any desired substrate with controlled assembly of elements i.e. of active areas with functionalized NPs. The laser beam is focused on a substrate/solution interface to decompose the precursor. In this paper, we use $\left[\left\{\mathrm{Au}_{10} \mathrm{Ag}_{12}\right.\right.$ $\left.\left.\left(\mathrm{C}_{2} \mathrm{Ph}\right)_{20}\right\} \mathrm{Au}_{3}\left(\mathrm{PPh}_{2}\left(\mathrm{C}_{6} \mathrm{H}_{4}\right)_{3} \mathrm{PPh}_{2}\right)_{3}\right]\left[\mathrm{PF}_{6}\right]$, a supramolecular complex, (hereinafter termed SMC), which is dissolved in acetophenone, dichloroethane and acetone to realize nanoalloys of $\mathrm{Au}-\mathrm{Ag}$ embedded in a carbonaceous matrix. ${ }^{5}$ The embedded nanoalloys show a narrow size distribution with unique properties. Among those properties we studied the 
localized surface plasmon resonance (LSPR) ${ }^{6}$ that depends on analytes of interest that attach to the functionalized NP surfaces and surface enhanced Raman spectroscopy (SERS), ${ }^{6}$ a method which detects the Raman signature of analytes for which a signal enhancement through NPs can occur on the $\mu$-chips so that even extremely low analyte concentrations can be detected. Both spectroscopy techniques were exploited to detect bio-agents such as blood and a hazardous substance, anthracene. The LID method can be used not only to synthesize $\mathrm{C}-\mathrm{Au}-\mathrm{Ag}$ NPs, but other metal NP compounds as well as $\mathrm{Cu}, \mathrm{Au}$ and $\mathrm{Ni}$ or semiconductor quantum dots. ${ }^{6}$

\section{The method and device}

Fig. 1 shows the LID process: the deposition of $\mathrm{C}-\mathrm{Au}-\mathrm{Ag}$ NPs on a transparent conductive oxide (TCO) layer (here: indium tin oxide-ITO) was carried out by a He-Cd laser ( $\mathrm{CW}, \lambda=325 \mathrm{~nm}$, $\left.P=1 \mathrm{~mW} \mathrm{~cm}{ }^{-2}\right)$ on a large $(3 \mathrm{~mm})$ or a small area $(20 \mu \mathrm{m})$ using an unfocussed or a focused laser beam, respectively ( $c f$. Fig. 1A, B). The focused laser beam was used to realize controlled elements of the $\mu$-devices (Fig. 1C) while the unfocussed laser can be used to realize m-layers. The choice of using HeCd UV laser was motivated by the strong absorption band of the SM complex at $328 \mathrm{~nm}$ (Fig. SI_1). $\hat{\dagger}^{6}$

Thus, an efficient light induced decomposition of the SM complex can be triggered, rather than uncontrolled thermal fragmentation. ${ }^{6} \mathrm{C}-\mathrm{Au}-\mathrm{Ag}$ NPs are formed as a result of transformation of the supramolecular complex under laser irradiation and subsequent agglomeration. As the laser irradiates the surface of the substrate, the $\mathrm{C}-\mathrm{Au}-\mathrm{Ag}$ nanoparticles are formed onto the surface of the substrate. Fig. 2A \& B show an optical image layout of a $\mu$-chip with $10 \times 10$ elements each assuming a diameter of $20 \mu \mathrm{m}$ with spacings of $100 \mu \mathrm{m}$ (center to center of two adjacent elements). Fig. 2C illustrates a colored SEM image of an individual element. The morphology for each element (i.e. density distribution of the $\mathrm{C}-\mathrm{Au}-\mathrm{Ag}$ NPs) can be controlled by the laser power profile along the laser radii.

\section{Chemistry}

Fig. 3 shows scanning electron micrograph (SEM) images of the deposited $\mathrm{C}-\mathrm{Au}-\mathrm{Ag}$ NPs of a single element after using the focused laser on different ITO/solution interfaces i.e. different solvents at identical concentrations $\left(5 \mathrm{mg} \mathrm{ml}^{-1}\right)$ : acetophenone, dichloroethane and acetone ( $c f$. Fig. 3A-C).

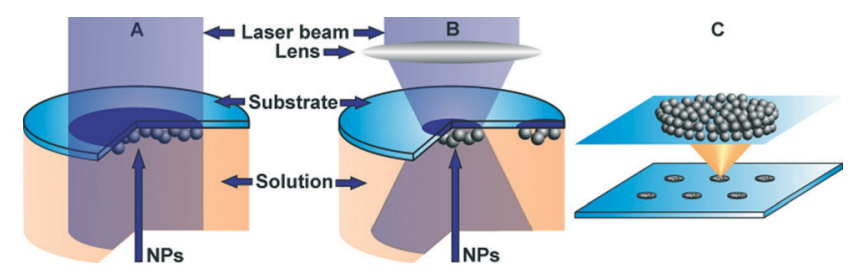

Fig. 1 The scheme of LID process: (A) deposition with unfocused laser beam, (B) deposition with focused laser beam; and (C) elements of the $\mu$-chip.

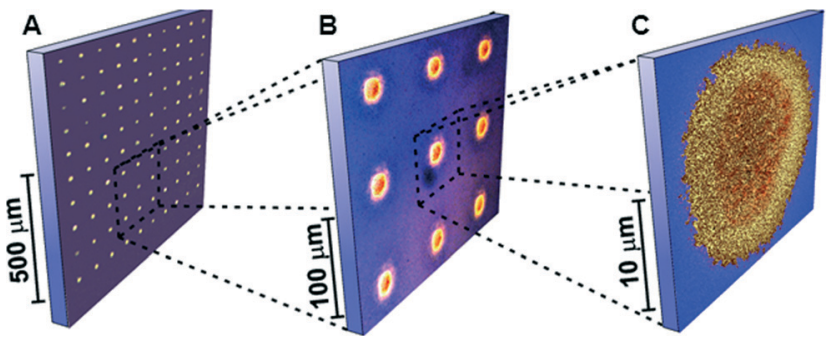

Fig. 2 Optical micrographs (A, B) of the $\mu$-chip consisting of $10 \times 10$ elements ( $20 \mu \mathrm{m}$ in diameter) with NP agglomerates of C-Au-Ag NPS as they turn out from our LID deposition technique. (C) Colored SEM image of single element.

As shown, the solvent can partially control the agglomeration size of the C-Au-Ag NP. Acetophenone, dichloroethane and acetone show agglomerated average sizes of $13.6 \pm 5 \mathrm{~nm}$, $17.3 \pm 2 \mathrm{~nm}$ and $19 \pm 5 \mathrm{~nm}$ (Fig. 3D-F). The different sizes of the deposited C-Au-Ag NPs are explained in terms of viscosity and density of the solvent. For example, acetone has the lowest viscosity $(0.3075 \mathrm{cP})$ and density $\left(0.7857 \mathrm{~g} \mathrm{~cm}^{-3}\right)$, which implies high diffusion rates of components, thus large agglomeration size in acetone is observed. ${ }^{7}$ The $\mathrm{Au}-\mathrm{Ag}$ combination provides large LSPR and controllable plasmon resonances, chemical stability and compatibility with various biomaterials.

The chemical composition of the agglomerated NPs was confirmed by energy dispersive X-ray spectra (EDX) as shown in Fig. SI_2.† The obtained data revealed the composition ratio of the deposited $\mathrm{C}-\mathrm{Au}-\mathrm{Ag}$ NPs to be 90:5:5 at.\%, regardless of the used solvent. It is worth noting that the SM complex consists of $286 \mathrm{C}$-atoms, $12 \mathrm{Au}$ atoms and $12 \mathrm{Ag}$ atoms, i.e. ratio of $92: 4: 4$ at.\%, which is consistent with the obtained composition. Precise chemical composition and size of the C-Au-Ag NPs was determined by high resolution transmission electron microscopy (HRTEM) as shown in Fig. 4.

The electron diffraction pattern in Fig. 4A confirms the presence of nanocrystalline $\mathrm{Ag}$ and $\mathrm{Au}$ NPs (the diffraction spacing is consistent with crystal data sets for $\mathrm{Au}$ and $\mathrm{Ag}$ as taken from the ICSD tables: ICSD 44837 for Ag and ICSD 44362 for Au). HRTEM images ( $c f$. Fig. 4B-E) show crystalline

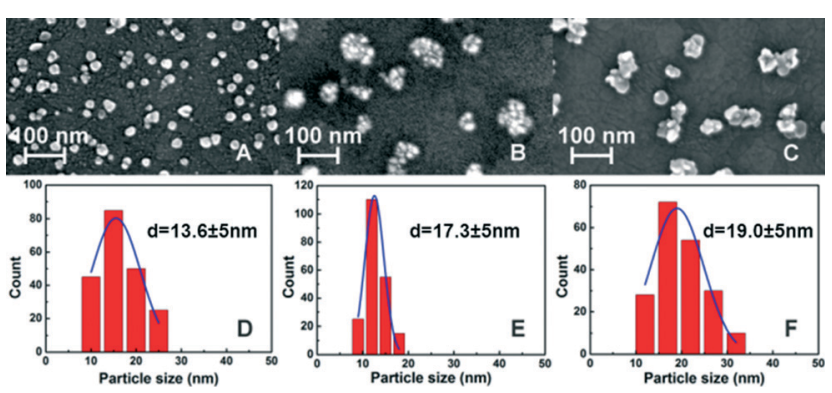

Fig. 3 HRSEM images of C-Au-Ag NPs deposited on a ITO from different solutions: (A) acetophenone, (B) dichloroethane and (C) acetone. The corresponding average particle agglomerates are $13.6 \pm$ $5 \mathrm{~nm}, 17.3 \pm 2 \mathrm{~nm}$ and $19 \pm 5 \mathrm{~nm}$, respectively (D-F). 

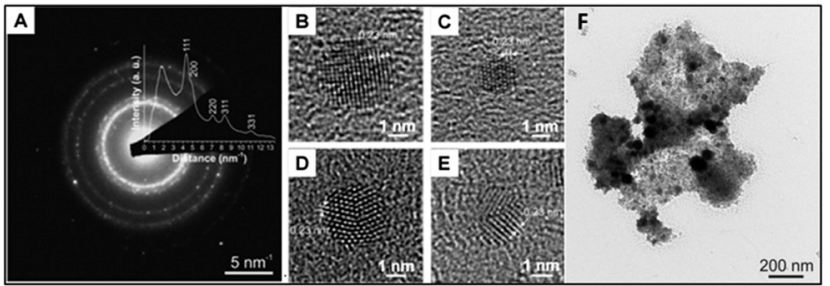

Fig. 4 (A) Electron diffraction of the nano-crystalline $\mathrm{Ag}$ and $\mathrm{Au}$. (A) An intensity profile of the electron diffraction pattern, obtained by radial averaging; $(B, C)$ HRTEM images of defect-free NPs in amorphous carbon matrix. (D, E) HRTEM of NP with stacking faults or twin boundaries. (F) TEM of agglomerated Au-Ag nanoalloys.

NPs 2-5 nm in diameter with partly extended defects such as stacking faults or twins. It should be mentioned that due to the identical crystal structure (face-centered cubic) of $\mathrm{Ag}$ and $\mathrm{Au}$ and almost the same lattice constants (Ag: $4.09 \AA$ А $\mathrm{Au}$ : $4.08 \AA$ ), it is difficult to say whether these NPs are an alloy or mixture of the two metals. An image of large NP agglomerates is shown in Fig. 4F. To get deeper insight into the local distribution of $\mathrm{Ag}$ and $\mathrm{Au}$ in the $\mathrm{C}-\mathrm{Au}-\mathrm{Ag}$ NPs, EDX mappings were performed (using a super-X EDS system from Bruker Instruments) and are illustrated in Fig. SI_3. $\dagger$ The results suggest a homogeneous $\mathrm{Au}-\mathrm{Ag}$ nanoalloy composition and are further supported by the absorption spectra (cf. Fig. SI_4†). The spectra show a single absorption peak between 400 and $500 \mathrm{~nm}$ which can be attributed to the plasmon resonance of a $\mathrm{Au}-\mathrm{Ag}$ nanoalloy. In case $\mathrm{Au}$ and $\mathrm{Ag}$ NPs would form separately, two separate plasmon peaks of $\mathrm{Au}$ and $\mathrm{Ag}$ would have been expected, which are however not observed experimentally. ${ }^{7}$ The nature of the carbon matrix was resolved by $\mu$-Raman spectroscopy ( $c f$. SI_4B) and showed $D$ and $\mathrm{G}$ bands, typical of carbonaceous materials, centered at $1350 \mathrm{~cm}^{-1}$ and $1587 \mathrm{~cm}^{-1}$, respectively, which are typical of amorphous and hydrogenated carbon (a-C:H). ${ }^{8,9}$ All SEM, TEM, absorption and Raman data are indicative of $\mathbf{A u}-\mathbf{A g}$ nanoalloys 2-5 $\mathrm{nm}$ diameter embedded in a carbonaceous matrix of $\mathrm{sp}^{2}$ hybridized carbon. There was no indication that the nanoalloy particle size can be controlled by solvents, solution concentrations or exposure time to the laser beam which is a clear signal for the NPs to form directly in the initial moments of exposure to laser light. The deposited $\mathrm{C}-\mathrm{Au}-\mathrm{Ag}$ NPs show an interesting Localized Surface Plasmon Resonance (LSPR) in the visible range that can be detected spectroscopically and can be exploited to excite vibrational modes in analytes of choice. We utilized the LSPR to enhance the Raman signal of bio-agents for a fast analysis of anthracene and human blood.

\section{Fast analysis of hazardous and biological substances}

Anthracene is a polycyclic aromatic hydrocarbon which is toxic with long-term negative effects on living creatures even at low concentrations ( $c f$. International Programme on Chemical Safety (IPCS)). To utilize the advantage of our $\mu$-chip elements to enhance LSPR to be exploited in a SERS experiment, we compare SERS signals of anthracene on the $\mu$-chip (red line) with SERS on a bare TCO layer without any NPs (blue line) and SERS on bulk crystals of anthracene (black line). Here we used two wavelengths for the SERS studies, namely $532 \mathrm{~nm}$ and $785 \mathrm{~nm} \cdot{ }^{10,11}$ As illustrated in Fig. 5, our $\mu$-chip elements enhanced the Raman signals even though highly dilute anthracene was used $\left(10^{-6} \mathrm{M}\right)$. Even more, the $\mu$-chip enhanced SERS signals of dilute anthracene were stronger than the signal of bulk crystals of anthracene with a high concentration $\left(10^{-2} \mathrm{M}\right)$. For highly dilute antracene on bare TCO with no signal enhancing NPs, no SERS signal could be determined. To explore the detection limit of the $\mu$-chip elements, we determined the SERS of a series of dilute anthracene concentrations ranging from $10^{-4} \mathrm{M}$ to $10^{-7} \mathrm{M}$ dissolved in Intralipid solution (which is a widely used bio-organic medium Fresenius Kabi Austria GmbH). Fig. 5B shows enhanced Raman signals as the antracene concentrations increased. In Fig. 5B the analyte concentration is shown as a function of the intensity of the pronounced Raman peak at $1400 \mathrm{~cm}^{-1}$.
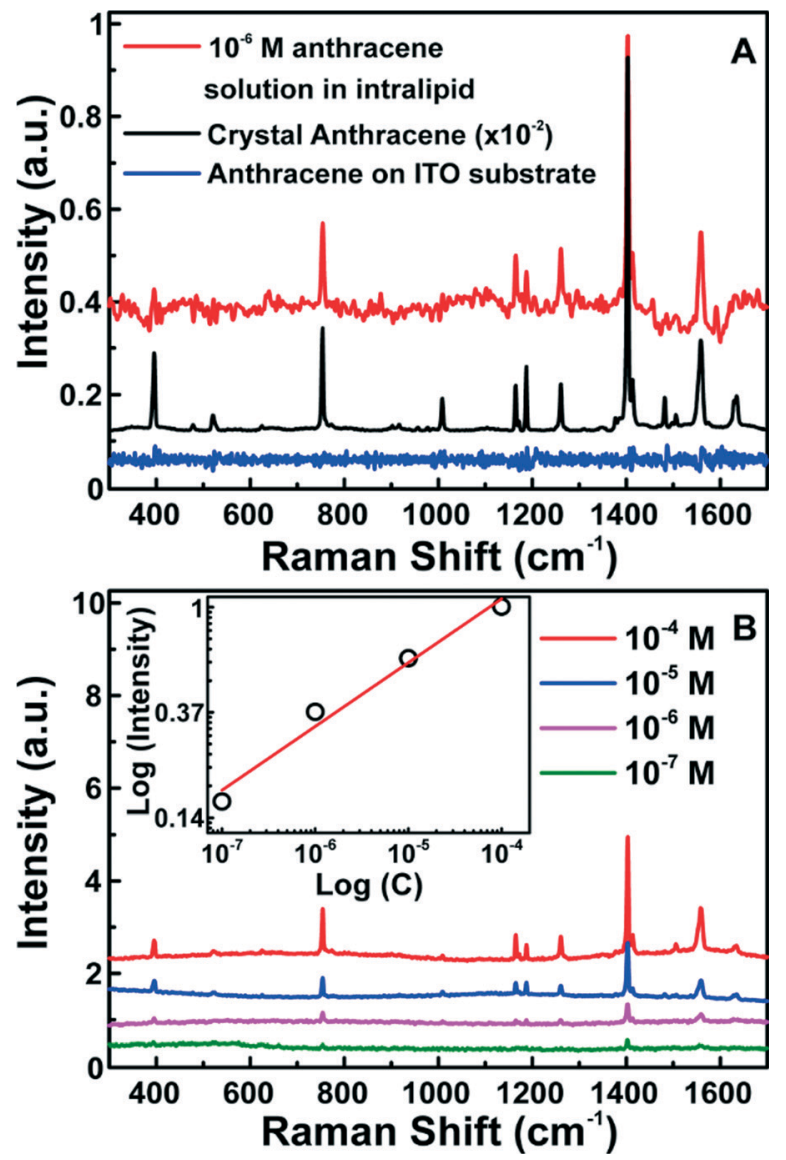

Fig. 5 (A) Raman spectrum of anthracene; (red line): low concentration $\left(10^{-6} \mathrm{M}\right)$ of anthracene on an element, (black line): bulk crystals of anthracene $\left(\times 10^{-2}\right)$ on an element, (blue line) reference sample of anthracene on black ITO i.e. no elements used. (B) Raman intensity of highly dilute solutions of anthracene in acetone deposited on element; (excited with $532 \mathrm{~nm}$ laser light). Inset: concentration dependence (molarity M) of the Raman intensity of the $1400 \mathrm{~cm}^{-1}$ peak of anthracene (note: logarithmic scale). 
This curve shows a linear correlation between the SERS signal intensity on the $\mu$-chip elements and the analyte concentration with an extrapolated detection limit in the range of $10^{-8}$ $10^{-9} \mathrm{M}$. This implies that our elements (especially the $\mathrm{Au}-\mathrm{Ag}$ functionality) support the detection of analytes by enhancing LSPs and may be, by encouraging the attachment of analytes on the $\mathrm{C}-\mathrm{Au}-\mathrm{Ag}$ NP surfaces, a speculation which will be further substantiated later in this paper. The $\mu$-chip elements support a highly sensitive analysis of analytes and can thus be used in sensors for bio-agents. Changing the $\mu$-chip's elements' properties such as the agglomeration size of the C-Au-Ag nanoalloys, the Raman signal enhancement can be further controlled as shown in Fig. SI_5.† The role of the carbonaceous shell of the $\mathrm{C}-\mathrm{Au}-\mathrm{Ag}$ NPs was also examined.

While the Au-Ag NPs of the $\mu$-chip elements are responsible for the LSPR excitation and thus for the signal enhancement in SERS, the carbonaceous shell can be used to enhance the adsorption of the analyte on the surface in an efficient way. ${ }^{12}$ To study the adsorption properties of the carbonaceous shell, we immersed the $\mu$-chip elements in a dilute analyte solution ( $2 \mathrm{~g}$ of human blood in a liter of deionized water) for different time frames from 0 to $20 \mathrm{~min}$ (cf. Fig. 6). The increase in the Raman intensity with longer immersion times indicates an effective adsorption of blood on the carbon shell. The efficient adsorption makes the $\mu$-chip an ideal device for SERS detection since the elements do not only support the Raman signal enhancement through the $\mathrm{Ag}-\mathrm{Au}$ nanoalloys but also support the analyte adsorption on the carbonaceous shell and makes the devices useful to detect ultra-small fractions in the analyte and low concentrations.

\section{Multiplex detection capacity}

For demonstration of the multiplex detection capacity of our $\mu$-chip, we measured the analytes separately and all together.

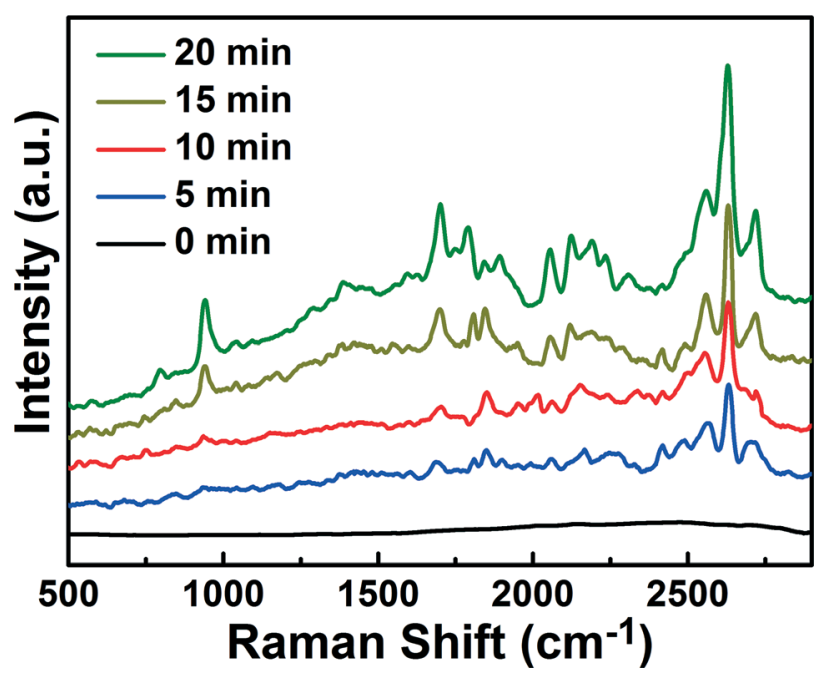

Fig. 6 Raman spectra of dilute human blood (contains blood plasma as well as red blood cells) in deionized water $\left(2 \mathrm{~g} \mathrm{l}^{-1}\right.$ blood, $\left.10^{-7} \mathrm{M}\right)$ measured at various immersion times ( 0 to $20 \mathrm{~min}$ ) on the same element.
Human blood solution (20 $\left.\mathrm{g} \mathrm{l}^{-1}\right)$ and anthracene solutions $\left(1.78 \times 10^{-3} \mathrm{~g} \mathrm{l}^{-1}\right)$ were used as analytes and were deposited separately onto two different $\mu$-chip elements, while a mixture of both solutions was prepared and analyzed as well. Fig. 7 shows Raman signals with $1 \mu$ drops of analytes to be dispensed using a micropipette (Lenpipet Black 1-10 $\mu$ l Thermo Scientific) while controlling spotting with a microscope. The first two elements (green and red) show typical Raman signals while the third element shows a superposition of the two individual Raman signatures ( $c f$. Fig. 7). This illustrates the capacity of the $\mu$-chip to detect various analytes at low concentrations and volumes. Moreover, the reproducibility of the $\mu$-chip was confirmed by measuring Raman signatures of the same analyte on different LID deposited elements (which are supposed to be identical in terms of LID procedures) by showing identical Raman spectra. Identical Raman spectra measured at different LID elements on the $\mu$-chip using identical analytes confirm the large reproducibility of the LID method and the robustness of the $\mu$-chip signals. (cf. Fig. SI_7). $\dagger$

\section{Summary}

In summary our results demonstrate a direct writing method (termed LID) using focused laser beams to deposit C-Au-Ag NPs from liquid precursors (a Au and Ag containing supramolecular complex, termed SMC) to fabricate fast and reliably $\mu$-chips with various elements, each of which having a diameter of several $\mu \mathrm{m}(20 \mu \mathrm{m})$ and containing $\mathrm{C}-\mathrm{Au}-\mathrm{Ag}$ NPs to enhance analyte fingerprints in optical detection methods such as SERS. The role of the carbon in the C-Au-Ag NPs was determined and enhanced adsorption capacity of analytes could be detected. The $\mu$-chip with $\mathrm{C}-\mathrm{Au}-\mathrm{Ag}$ NPs as active elements permits the detection of highly dilute analytes with $10^{-8}-10^{-9} \mathrm{M}$ and remains stable throughout the analysis i.e. does not decompose or dissolve in organic analyte solutions. The simplicity of the LID method for the fast and controlled composite NP deposition is remarkable to realize highly sensitive $\mu$-chips e.g. SERS which have the potential to be used

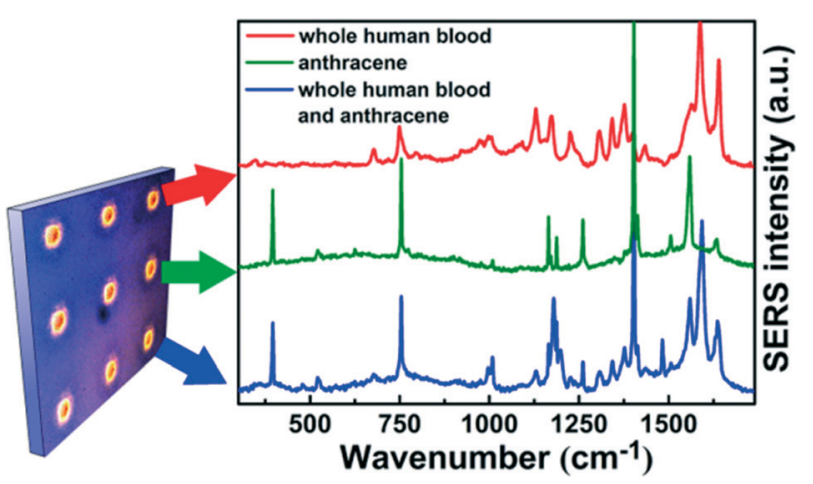

Fig. 7 Raman spectrum of human blood in deionized water $\left(10^{-6} \mathrm{M}\right)$, (red line), Raman spectrum of anthracene in water $\left(10^{-6} \mathrm{M}\right)$, (green line), and human blood/anthracene solution mixture (1:1 vol\%), (blue line). 
even in commercial setups with technological relevance. Our $\mu$-chips may be adopted for sensitive analyte detection in areas of bio-medical screening, detection of hazardous materials in homeland security and environmental monitoring missions.

\section{Experimental section}

\section{Materials}

NPs were deposited onto the surface of a TCO from solutions of a supramolecular complex termed SMC, $\left[\left\{\mathrm{Au}_{10} \mathrm{Ag}_{12^{-}}\right.\right.$ $\left.\left.\left(\mathrm{C}_{2} \mathrm{Ph}\right)_{20}\right\} \mathrm{Au}_{3}\left(\mathrm{PPh}_{2}\left(\mathrm{C}_{6} \mathrm{H}_{4}\right)_{3} \mathrm{PPh}_{2}\right)_{3}\right]\left[\mathrm{PF}_{6}\right]$, throughout the text by a one-step laser-induced chemical liquid phase deposition (LID) method. ${ }^{6}$ This SMC was synthesized according to a published procedure. ${ }^{5}$ The solutions for LID based NP deposition were prepared by dissolving $5 \mathrm{mg}$ of SMC in $1 \mathrm{ml}$ of solvent (acetone, dichloroethane and acetophenone of analytical grade purity). To remove the undissolved components, the solution was centrifuged at $10000 \mathrm{rpm}$ for $5 \mathrm{~min}$. Then an aliquot of solution was placed into a cuvette, covered by the substrate of choice. The solution covered substrate is subsequently illuminated by a focused or defocussed laser beam for laser induced deposition (LID).

\section{Characterization LID method}

The preparation of $\mu$-chips that can serve as SERS substrates was carried out as follows: the transparent TCO/glass substrate was fixed on a cuvette containing the solution with the SMC complex. The UV laser beam was focused $(20 \mu \mathrm{m}$ in diameter at $1 / \mathrm{e}^{2}$ intensity with $10 \mathrm{sec}$ exposure time) through the substrate at the substrate-solution interface ( $c f$. Fig. 1b). As a result of laser irradiation, spots or elements with $\sim 20 \mu \mathrm{m}$ sized deposits composed of $\mathrm{C}-\mathrm{Au}-\mathrm{Ag}$ NP composites were realized ( $c f$. Fig. 1c). For the direct writing of $\mathrm{C}-\mathrm{Au}-\mathrm{Ag}(90: 5: 5)$ we used the aforementioned SMC complex and dissolved it in various solvents and deposited on a transparent conductive oxide (TCO) layer (here: Al-doped $\mathrm{ZnO}$ and indium-tinoxide were used) on glass substrates. The $\mu$-chip contains an array of $10 \times 10$ elements that show a pitch of $\sim 100 \mu \mathrm{m}$ (measuring between the centers of two adjacent spots). One of those $\mu$-chips could be prepared within $20 \mathrm{~min}$. Finally, even these $\mu$-chips were washed in acetone and dried at $40{ }^{\circ} \mathrm{C}$ for $50 \mathrm{~min}$. Analyte delivery: A PLI-10 Pico-Injector reliably delivers ejections from femtoliters to nanoliters through nano-pipettes by applying a regulated pressure for a digitally set period of time and micro-positioner system. This can be used in labs for express analysis of ultra-low volumes. The minimal possible volume of the analyte solution for analysis with a $\mathrm{C}-\mathrm{Au}-\mathrm{Ag} \mathrm{m}$-chip is determined by the volume required for total spot coating and can be as small as $40 \mathrm{pl}$ for a $20 \mathrm{~mm}$ spot. The pl volume was estimated from test experiments with $1 \mu \mathrm{L}$ drops for estimation of liquid spreading on the NP surface. A $40 \mathrm{pl}$ drop of analyte allows total coating of the spot with NPs with a diameter of $\sim 20 \mathrm{~mm}$. The elements were not washed after SERS measurements to eliminate the impact of the phys-adsorbed molecules of the solvent. A $1 \mu \mathrm{L}$ drop covers several elements, and we used it for demonstration of the reproducibility of the LID method and the robustness of the $\mu$-chip signals (Fig. SI_7b $†$ ). For a single element we used the Pico-Injector. $\boldsymbol{\mu}$-Chip: the design demonstrates the possibility of deposition of elements with precise control of the position and creation of a $\mu$-chip with high density of elements. That allows investigation of a big number of analytes. The design of the $\mu$-chip can be optimized for the exact task. The laser spot for the Raman and SERS measurements was about $2.5 \mu \mathrm{m}$ ( $\times 50$ objective) and was focused to a single element (which is about $20 \mu \mathrm{m}$ ). We used the focusing system of a Senterra Raman spectrometer. SEM: the images of the deposited particles were obtained with a field emission scanning electron microscope (FE-SEM, Carl Zeiss AG, Merlin). Images were obtained at $8 \mathrm{kV}$ acceleration voltage in secondary electron detection mode with a standard EverhartThornley detector. Helium ion microscopy images were obtained using a helium ion microscope (Carl Zeiss AG, Orion) at $35 \mathrm{kV}$ acceleration voltage in secondary electron detection mode with a standard Everhart-Thornley detector. TEM: conventional transmission electron microscopy (CTEM) and high-resolution TEM (HRTEM) were performed using a $\operatorname{Titan}^{3}$ 80-300 (FEI Company) equipped with a Schottky emitter, an image-side Cs-corrector (CEOS) and an energy dispersive X-ray spectroscopy (EDXS) detector. The microscope was operated at $300 \mathrm{kV}$ acceleration voltage. EDX spectroscopy was used in STEM mode to obtain local chemical information on the nanoscale. Electron diffraction was performed using a Philips CM30 TWIN/STEM, equipped with a $\mathrm{LaB}_{6}$ filament and operated at $300 \mathrm{kV}$ acceleration voltage. Electron diffraction patterns were studied by using the software JEMS (version 3.6608U2011), incorporating the crystal data from the Inorganic Crystal Structure Database (ICSD). TEM images and diffraction patterns were acquired with slow scan charge coupled device (CCD) cameras from TVIPS (Munich, Germany) and Gatan (USA), having image sizes of $1024 \times 1024$ and $2048 \times 2048$ pixels, respectively. The analysis of TEM images was carried out by using the commercial software DigitalMicrographTM (Gatan, USA) and a free available software ImageJ (version 1.45S). A further detailed analysis was carried out using a Cs-probe-corrected FEI Titan 80-300. Moreover, Z-contrast high angle annular dark field (HAADF) STEM imaging was used. This FEI Titan microscope is equipped with a Super-X EDS (ChemiSTEM Technology) system for ultimate EDX resolution. These measurements could provide high resolution elemental distribution maps. UV/VIS/ NIR: the absorption spectra of the deposited NPs were measured with a Precision Spectrophotometer Lambda 1050 UV/VIS/NIR (Varian). Measurements of the deposited NP absorption spectra were carried out with an integrating sphere in a spectral range from $350 \mathrm{~nm}$ to $600 \mathrm{~nm}$. Micro-RAMAN spectroscopy: the SERS measurements were performed using a Raman spectrometer (Senterra) equipped with a confocal microscope. The SERS spectra were recorded at room temperature using lasers with the following parameters: $\lambda_{\mathrm{ex}}=532 \mathrm{~nm}$ 
at a power of $0.2 \mathrm{~mW}$ and $\lambda_{\mathrm{ex}}=785 \mathrm{~nm}$ at a power of $1 \mathrm{~mW}$. The lasers were focused with a spot size of $10^{-6} \mathrm{~cm}^{2}$. The spectra were collected over ten seconds in back reflection geometry through a $50 \times 0.7 \mathrm{NA}$ objective. The reflected signal was focused onto a thermo-electrically cooled CCD array. The experimental parameters were kept the same for all SERS experiments.

\section{Acknowledgements}

The authors greatly appreciate financial support of SaintPetersburg State University under the research grant 0.37.169.2014, the University of Eastern Finland (strategic funding - Russian-Finnish collaborative project) and RFBR grants 13-03-12411 and 14-02-31031 mol_a and the GermanRussian Interdisciplinary Science Center (G-RISC) funded by the German Federal Foreign Office via the German Academic Exchange Service (DAAD) (proposals C-2012b-4, C-2013a-3). The authors are grateful to Anna Carlsson, a Sr. Application Specialist at FEI Company for TEM images presented in Fig. SI_3.† The Raman and UV/VIS absorption spectra were measured at the Center for Optical and Laser Materials Research, SaintPetersburg State University. A part of TEM and all SEM analysis was carried out at the Interdisciplinary Resource Center for Nanotechnology, Saint-Petersburg State University. M.Y.B gratefully acknowledges the Max-Planck Society for a Post-Doctoral fellowship. S.H.C. acknowledges the German Research Foundation (DFG) for financial support within the GRK1896, the Cluster of Excellence "Engineering of Advanced Materials" EAM at Friedrich-Alexander-University Erlangen-Nürnberg. Moreover, S.H.C. received funding from the European Commission within the Seventh Framework Programme [FP7/2007-2013] under grant agreement nr. 280566 - UnivSEM.

\section{References}

1 (a) E. Dovgolevsky, G. Konvalina, U. Tisch and H. Haick, J. Phys. Chem. C, 2010, 114, 14042-14049; (b) G. Peng, M. Hakim, Y. Y. Broza, S. Billan, R. Abdah-Bortnyak, A. Kuten, U. Tisch and H. Haick, Br. J. Cancer, 2010, 103, 542-551; (c) U. Tisch and H. Haick, Rev. Chem. Eng., 2010, 26, 171-179; (d) U. Tisch and H. Haick, MRS Bull., 2010, 35, 797-803; (e) M. Y. Bashouti, R. T. Tung and H. Haick, Small, 2009, 23, 2761-2767.

2 (a) M. Y. Bashouti, A. S. de la Zerda, D. Geva and H. Haick, J. Phys. Chem. C, 2014, 118, 1903-1909; (b) J. M. R. Tan, J. J. Ruan, H. K. Lee, I. Y. Phangb and X. Y. Ling, Phys. Chem. Chem. Phys., 2014, 16, 26983-26990; (c) Y. Hanada, K. Sugioka and K. Midorikawa, Lab Chip, 2012, 12, 3688-3693; (d) B.-B. Xu, Y.-L. Zhang, H. Xia, W.-F. I. Dong, H. Dingc and H.-B. Sun, Lab Chip, 2013, 13, 1677-1690; (e) Z. Q. Zhy, Z. D. Yan, P. Zhan and Z. L. Wang, Sci. China: Phys., Mech. Astron., 2013, 56, 1806-1809.

3 (a) A. G. Shen, L. F. Chen, W. Xie, J. C. Hu, A. Zeng, R. Richards and J. M. Hu, Adv. Funct. Mater., 2010, 20, 969-975; (b) S. C. Tang, S. Vongehr, G. R. He, L. Chen and X. K. Meng, J. Colloid Interface Sci., 2012, 375, 125-133; (c) S. C. Tang, S. Vongehr and X. K. Meng, J. Mater. Chem., 2010, 20, 5436-5445.

4 S. J. Henley, P. C. P. Watts, N. Mureau and S. R. P. Silva, Appl. Phys. A: Mater. Sci. Process., 2008, 93, 875-879.

5 (a) I. O. Koshevoy, A. J. Karttunen, J. R. Shakirova, A. S. Melnikov, M. Haukka, S. P. Tunik and T. A. Pakkanen, Angew. Chem., Int. Ed., 2010, 49, 8864-8866; (b) I. O. Koshevoy, A. J. Karttunen, S. P. Tunik, M. Haukka, S. I. Selivanov, A. S. Melnikov, P. Y. Serdobintsev and T. A. Pakkanen, Organometallics, 2009, 28, 1369-1376.

6 (a) A. Manshina, T. Ivanova and A. Povolotskiy, Laser Phys., 2010, 20, 1532-1536; (b) A. A. Man'shina, A. V. Povolotskiy, T. Y. Ivanova, A. V. Kurochkin, Y. S. Tver'yanovich, D. Kim, M. Kim and S. Kwon, Glass Phys. Chem., 2007, 33, 209-213; (c) A. A. Manshina, A. V. Povolotskiy, T. Y. Ivanova, Y. S. Tver'yanovich, S. P. Tunik, D. Kim, M. Kim and S. C. Kwon, Appl. Phys. A: Mater. Sci. Process., 2007, 89, 755-759; (d) A. A. Manshina, A. V. Povolotskiy, A. V. Povolotskaya, T. Y. Ivanova, I. O. Koshevoy, S. P. Tunik, M. Suvanto and T. A. Pakkanen, Surf. Coat. Technol., 2012, 206, 3454-3458; (e) A. Povolotskiy, A. Povolotckaia, Y. Petrov, A. Manshina and S. Tunik, Appl. Phys. Lett, 2013, 103; $(f)$ B. B. Xu, Y. L. Zhang, R. Zhang, L. Wang, X. Z. Xiao, H. Xia, Q. D. Chena and H. B. Sun, J. Mater. Chem. C, 2013, 1, 4699-4704; (g) K. Kordas, L. Nanai, G. Galbacs, A. Uusimaki, S. Leppavuori and K. Bali, Appl. Surf. Sci., 2000, 158, 127-133; (h) G. M. Herrera, A. C. Padilla and S. P. Hernandez-Rivera, Nanomaterials, 2013, 3, 158-172.

7 M. P. Mallin and C. J. Murphy, Nano Lett., 2002, 2, 1235-1237; P. S. Shah, S. Husain, K. P. Johnston and B. A. Korgel, J. Phys. Chem. B, 2002, 106, 12178.

8 A. C. Ferrari and J. Robertson, Phys. Rev. B: Condens. Matter Mater. Phys., 2000, 61, 14095-14107.

9 (a) S. H. Hong and J. Winter, J. Appl. Phys., 2005, 98, 124304; (b) P. K. Chu and L. H. Li, Mater. Chem. Phys., 2006, 96, 253-277.

10 H. Shinohara, Y. Yamakita and K. Ohno, J. Mol. Struct., 1998, 442, 221-234.

11 M. Moskovits, Rev. Mod. Phys., 1985, 57, 783-826.

12 (a) C. Moreno-Castilla, Carbon, 2004, 42, 83-94; (b) X. M. Sun and Y. D. Li, Angew. Chem., Int. Ed., 2004, 43, 597-601. 